\title{
Electric-Field Control of a Single-Atom Polar Bond
}

\author{
Omidian, M.; Leitherer, S.; Néel, N.; Brandbyge, M.; Kröger, J.
}

Published in:

Physical Review Letters

Link to article, DOI:

10.1103/PhysRevLett.126.216801

Publication date:

2021

Document Version

Publisher's PDF, also known as Version of record

Link back to DTU Orbit

Citation (APA):

Omidian, M., Leitherer, S., Néel, N., Brandbyge, M., \& Kröger, J. (2021). Electric-Field Control of a Single-Atom Polar Bond. Physical Review Letters, 126(21), [216801]. https://doi.org/10.1103/PhysRevLett.126.216801

\section{General rights}

Copyright and moral rights for the publications made accessible in the public portal are retained by the authors and/or other copyright owners and it is a condition of accessing publications that users recognise and abide by the legal requirements associated with these rights.

- Users may download and print one copy of any publication from the public portal for the purpose of private study or research.

- You may not further distribute the material or use it for any profit-making activity or commercial gain

- You may freely distribute the URL identifying the publication in the public portal

If you believe that this document breaches copyright please contact us providing details, and we will remove access to the work immediately and investigate your claim. 


\title{
Electric-Field Control of a Single-Atom Polar Bond
}

\author{
M. Omidian $\odot,{ }^{1}$ S. Leitherer $\odot,{ }^{2, *}$ N. Néel $\odot,{ }^{1}$ M. Brandbyge $\odot,{ }^{2}$ and J. Kröger ${ }^{1, \dagger}$ \\ ${ }^{1}$ Institut für Physik, Technische Universität Ilmenau, D-98693 Ilmenau, Germany \\ ${ }^{2}$ Center of Nanostructured Graphene, Department of Physics, Technical University of Denmark, DK-2800 Kongens Lyngby, Denmark
}

(Received 4 December 2020; revised 7 March 2021; accepted 12 April 2021; published 25 May 2021)

\begin{abstract}
We expose the polar covalent bond between a single $\mathrm{Au}$ atom terminating the apex of an atomic force microscope tip and a $\mathrm{C}$ atom of graphene on $\mathrm{SiC}(0001)$ to an external electric field. For one field orientation, the $\mathrm{Au}-\mathrm{C}$ bond is strong enough to sustain the mechanical load of partially detached graphene, while for the opposite orientation, the bond breaks easily. Calculations based on density-functional theory and nonequilibrium Green's function methods support the experimental observations by unveiling bond forces that reflect the polar character of the bond. Field-induced charge transfer between the atomic orbitals modifies the polarity of the different electronegative reaction partners and the $\mathrm{Au}-\mathrm{C}$ bond strength.
\end{abstract}

DOI: 10.1103/PhysRevLett.126.216801

Exploring the impact of electric fields on the modification of chemical-bond strengths is important for, e.g., electron transport across contacts in miniaturized devices and circuits [1]. Previously, the bond strength was controlled by modifying the number of covalent bonds atom by atom with the tip of a scanning tunneling microscope (STM), giving rise to characteristic changes in the overall junction conductance [2,3]. Electric-field effects and electrostatic forces have, moreover, been identified as notable ingredients for interpreting the contrast mechanism of an atomic force microscope (AFM) tip terminated by a single CO molecule [4-10].

Given the relevance of individual chemical bonds and electrostatic effects in junctions at the ultimate scale, it is desirable to explore the response of a single bond to an external electric field. The combined experimental and theoretical work presented here provides direct evidence for the influence of an electric field on the strength of a polar covalent bond between two atoms. An AFM is used to form and break in a controllable manner a bond between the $\mathrm{Au}$ atom terminating the AFM tip and a $\mathrm{C}$ atom of graphene on $\mathrm{SiC}(0001)$. The electric field is supplied by applying a voltage across the atomic junction. An electric field pointing from the $\mathrm{C}$ to the $\mathrm{Au}$ atom (positive sample voltage $V_{\text {sample }}$ ) yields a strong $\mathrm{Au}-\mathrm{C}$ bond that enables the detachment of graphene from the surface in the course of tip retraction; the opposite field direction (negative $V_{\text {sample }}$ ), in contrast, induces a weak and easily breakable $\mathrm{Au}-\mathrm{C}$ bond. Density-functional calculations including biased electrodes trace these observations to short-range bond forces that result from the polar covalent $\mathrm{Au}-\mathrm{C}$ chemical bond, whose strength is determined by the field-dependent charge allocation at the atoms.

Figure 1 summarizes the first part of the novel experimental results for clean [Figs. 1(a)-1(c)] and Li-intercalated [Figs. 1(d)-1(f)] graphene on $\mathrm{SiC}(0001)$ (see
Supplemental Material [11]). Clean graphene [Fig. 1(a)] yields STM images that are characterized by the previously reported $6 \times 6$ superlattice with a spatial period of $1.72 \pm 0.17 \mathrm{~nm}[32,33]$ and the graphene lattice where the honeycomb cells are separated by $0.23 \pm 0.02 \mathrm{~nm}$, in agreement with expectations $(0.246 \mathrm{~nm})$ [34]. For Liintercalated graphene, the $6 \times 6$ superstructure is absent [Fig. 1(d)], signaling the efficient migration of Li through the Bernal-stacked graphene and $\mathrm{C}$ buffer layer on $\mathrm{SiC}(0001)$ $[12,35,36]$.

Approaching the AFM tip toward the graphene lattice with $V_{\text {sample }}=-700 \mathrm{mV}$ and simultaneously recording the resonance frequency change $\Delta f$ of the oscillating tuning fork leads to the distance-dependent dataset referred to as $\Delta f_{\downarrow}(z)$ ( $z$, tip displacement) in the following and depicted as the upper solid line in Fig. 1(b). The associated vertical force $[37,38] F_{\downarrow}(z)$ appears as the lower solid line. Care has been taken to show well-posed force data by appropriately adjusting the maximum probed distance range with respect to the position of inflection points in the extracted force [39]. The minimum signals the point of maximum attraction. Beyond contact, the evolution of $F_{\downarrow}(z)$ deviates from the expected Lennard-Jones behavior, which would exhibit a steep increase due to Pauli repulsion. Most likely, atomic relaxations of the junction geometry are the cause for the deviations. Retraction of the AFM tip gives rise to $\Delta f_{\uparrow}(z)$ and $F_{\uparrow}(z)$ data, which are depicted as dashed lines in Fig. 1(b). Obviously, approach and retraction data virtually coincide; that is, $\Delta f_{\downarrow}(z) \approx \Delta f_{\uparrow}(z)$ and $F_{\downarrow}(z) \approx F_{\uparrow}(z)$. Using the opposite polarity of $V_{\text {sample }}$ [Fig. 1(c)] leads to a significantly different behavior of $\Delta f_{\downarrow}(z)$ and $\Delta f_{\uparrow}(z)$. Rather than reaching a well-defined minimum, $\Delta f_{\downarrow}$ abruptly changes its slope (see Supplemental Material [11]). Commencing the tip retraction at point $A, \Delta f_{\uparrow}(z)$ data do not reproduce $\Delta f_{\downarrow}(z)$ in the contact region. The $\Delta f_{\uparrow}$ trace intersects $\Delta f_{\downarrow}$ at point $R$ 
(a)

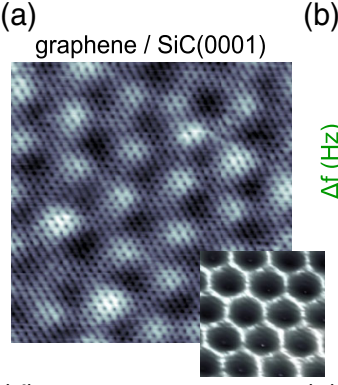

(d)

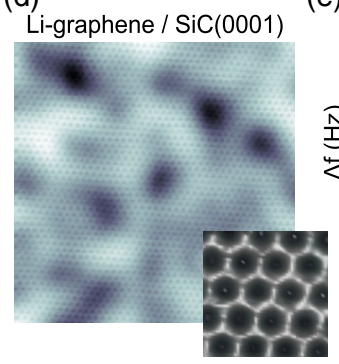

(e) (b)
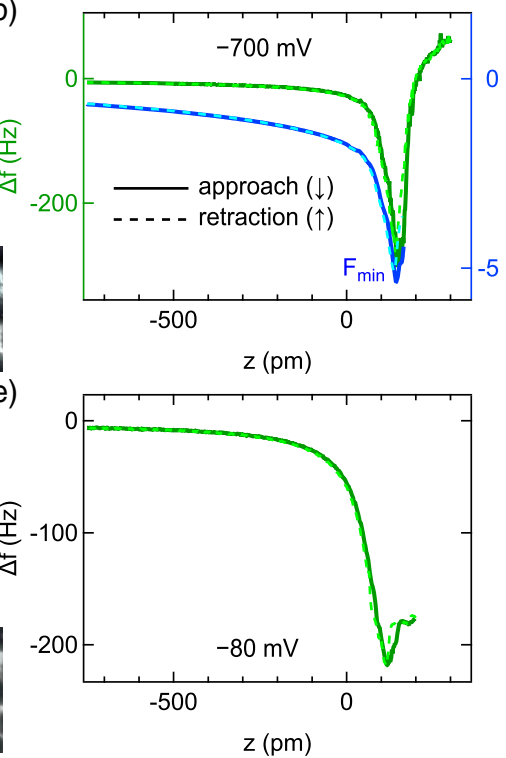

(c)

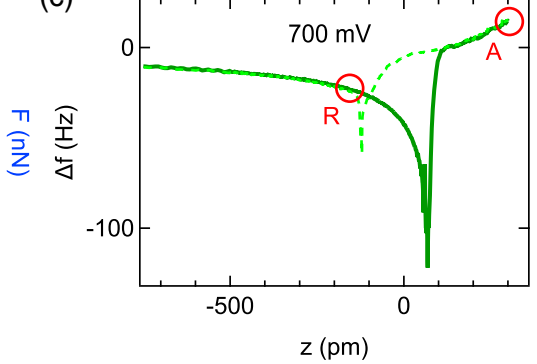

(f)

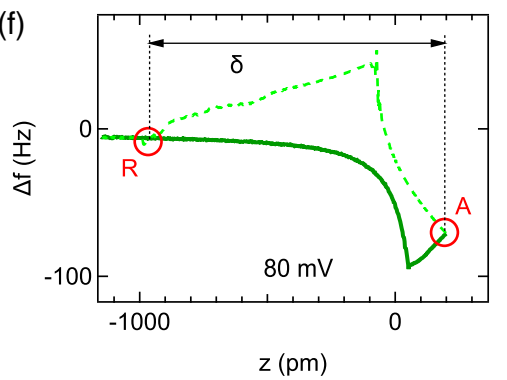

FIG. 1. (a) Constant-current STM image of clean graphene [bias voltage: $-10 \mathrm{mV}$; tunneling current: $55 \mathrm{pA}$; size, $9.7 \times 9.7 \mathrm{~nm}^{2}$; the gray scale ranges from 0 (black) to $67 \mathrm{pm}$ (white)] with close-up view of the graphene lattice [1 mV, $33 \mathrm{pA}, 0$ (black)-100 pm (white)]. (b) $\Delta f(z)$ for clean graphene, $V_{\text {sample }}=-700 \mathrm{mV}$ (approach, from left to right, upper solid line; retraction, from right to left, upper dashed line). The vertical force $F$ is displayed as the lower solid (approach) and dashed (retraction) line ( $F_{\min }$, point of maximum attraction). (c) $\Delta f(z)$ for clean graphene, $V_{\text {sample }}=700 \mathrm{mV}$ ( $A$, start of tip retraction; $R$, intersection of $\Delta f_{\downarrow}$ and $\Delta f_{\uparrow}$ data). (d) Like (a) for Li-intercalated graphene [100 mV, $51 \mathrm{pA}, 10 \times 10 \mathrm{~nm}^{2}, 0$ (black)-81 pm (white); close-up view, $100 \mathrm{mV}, 50 \mathrm{pA}, 0$ (black)-32 pm (white)]. (e) $\Delta f(z)$ for Li-intercalated graphene, $V_{\text {sample }}=-80 \mathrm{mV}$. (f) Like (e), $V_{\text {sample }}=80 \mathrm{mV}$. The loop width of $\Delta f(z)$ is $\delta$. In (b), (c) and (e),(f), $z=0 \mathrm{pm}$ is defined as the tip position prior to deactivating the feedback control and retracting the tip into the tunneling range $(z<0 \mathrm{pm})$.

before coinciding with $\Delta f_{\downarrow}(z)$ for further retraction, giving rise to a $\Delta f$ loop.

A similar trend of $\Delta f_{\downarrow}$ and $\Delta f_{\uparrow}$ was observed for $\mathrm{Li}-$ intercalated graphene [Fig. 1(d)]; i.e., $\Delta f_{\downarrow}(z)$ and $\Delta f_{\uparrow}(z)$ are essentially identical for $V_{\text {sample }}<0 \mathrm{~V}$ [Fig. 1(e)] and strongly deviate from each other for $V_{\text {sample }}>0 \mathrm{~V}$ [Fig. 1(f)]. The loop width spanned by the distance between $A$ and $R, \delta=z_{A}-z_{R}$, is, however, larger for Li-intercalated graphene than for its pristine counterpart.

The presented contact experiments are reproducible. Subsequent approach-retraction cycles using the same tip, $V_{\text {sample }}$, and contact site yield virtually identical $\Delta f(z)$ data and leave the structural integrity of tip and sample invariant [11]. Therefore, forming and breaking the covalent tip-graphene bond is reversible. Moreover, the current evolution $I(z)$ across the junction exhibits a consistent loop behavior [11].

Before discussing the $V_{\text {sample }}$ dependence of the $\Delta f$ loop, a tentative interpretation of the experimental observations shall be offered here and corroborated below by the simulations. It seems that the chemical bond formed upon tip approach at $V_{\text {sample }}>0 \mathrm{~V}$ is strong enough to locally detach the graphene sheet upon tip retraction. Therefore, the $\Delta f_{\downarrow}$ data necessarily differ from $\Delta f_{\uparrow}$ for those distances where the graphene sheet is partially attached to the tip. The point where the $\Delta f(z)$ loop closes $[R$ in
Figs. 1(c) and 1(f)] would then correspond to the release of the lifted graphene. At $V_{\text {sample }}<0 \mathrm{~V}$, in contrast, $\Delta f_{\downarrow}$ and $\Delta f_{\uparrow}$ nearly coincide; i.e., the chemical bond formed between the tip and graphene is weak and easily broken by tip retraction-the graphene sheet remains on the surface and impedes the evolution of a $\Delta f$ loop.

To further characterize the $\Delta f$ behavior upon tip approach and retraction and its dependence on the $V_{\text {sample }}$ polarity, several other aspects were explored and are presented as the second part of the novel experimental results in Fig. 2. Figures 2(a) and 2(b) compare $\delta\left(V_{\text {sample }}\right)$ for clean [Fig. 2(a)] and Li-intercalated [Fig. 2(b)] graphene for a variety of tips. The different tips are characterized by the magnitude of $F_{\min }$. Repeatedly performed field emission on and indentations into a Au substrate presumably cover the PtIr tip apex with $\mathrm{Au}$ and lead to different macroscopic tip shapes. Therefore, the long-range van der Waals interaction between tip and surface is altered, which is reflected by $F_{\min }$. Both samples exhibit an asymmetric evolution of $\delta$ with the sign of $V_{\text {sample }}$. While $\delta$ vanishes for $V_{\text {sample }} \leq 0 \mathrm{~V}$, it starts to increase monotonically for $V_{\text {sample }}>0 \mathrm{~V}$. Moreover, $\delta$ stays comparably low for clean graphene. At $V_{\text {sample }}=1 \mathrm{~V}, \delta$ is still lower than $750 \mathrm{pm}$ [Fig. 2(a)]. For Li-intercalated graphene and a tip with similar force minimum $F_{\min }$ as in the case of clean graphene, $\delta$ adopts nearly $2000 \mathrm{pm}$ already at 

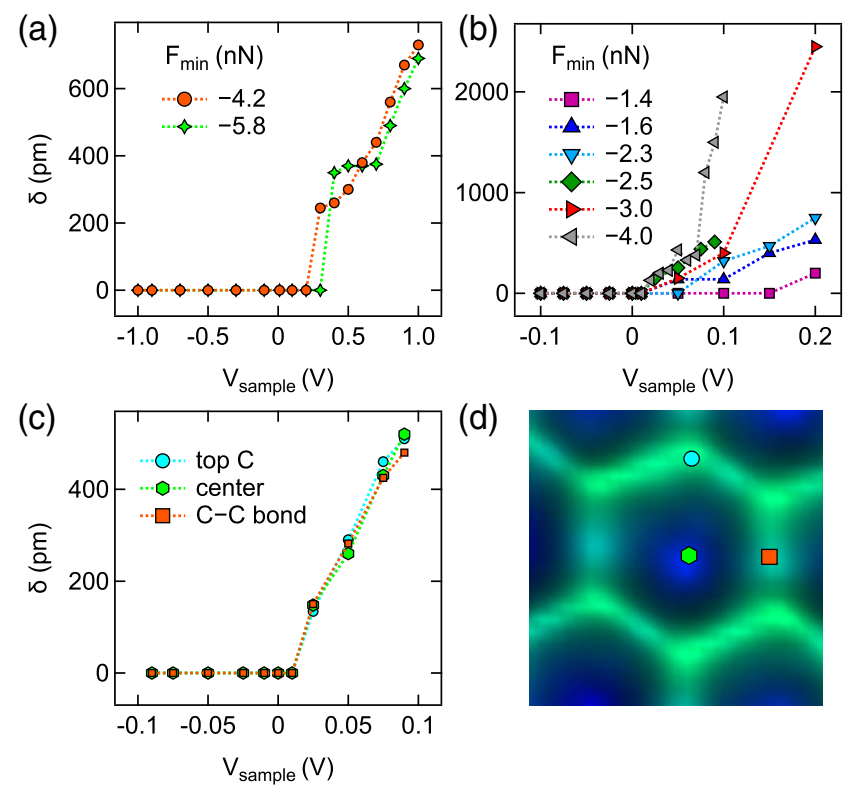

(d)

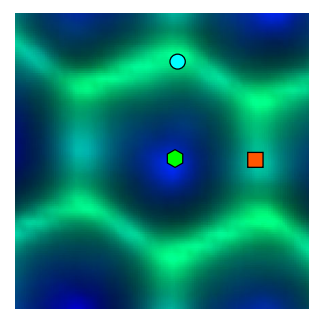

FIG. 2. (a) $\delta\left(V_{\text {sample }}\right)$ for clean graphene. (b) Like (a) for Li-intercalated graphene. Different tips in (a) and (b) are characterized by $F_{\text {min }}$. (c) Site dependence of $\delta\left(V_{\text {sample }}\right)$ for Li-intercalated graphene. (d) Constant-height $\Delta f$ map $\left[0.47 \times 0.47 \mathrm{~nm}^{2},-8.7\right.$ (dark) $-7.5 \mathrm{~Hz}$ (bright) $]$ of a graphene honeycomb cell.

$V_{\text {sample }}=0.1 \mathrm{~V}$ [Fig. 2(b)]. This effect is most likely caused by graphene being in its quasifree state, which facilitates its detachment from the surface.

For the Li-intercalated sample, $\delta\left(V_{\text {sample }}\right)$ data obtained are collected in Fig. 2(b). A clear trend is visible. An increase of $\left|F_{\min }\right|$ entails a larger slope of $\delta$ for $V_{\text {sample }}>0 \mathrm{~V}$; i.e., $\delta$ adopts large values already at low $V_{\text {sample }}$. This observation is plausible because the longrange van der Waals force acts as an additional background attraction and assists in lifting the graphene sheet [40-42]. The strength of the covalent tip-graphene bond, however, is determined by the short-range bond force, as clarified by the calculations below.

In a different set of experiments, the graphene lattice site dependence of the characteristic $\Delta f$ behavior was explored. To this end, the high symmetry points of the honeycomb cell [a $\mathrm{C}$ atom, a $\mathrm{C}-\mathrm{C}$ bond, and a honeycomb center, Fig. 2(d)] were scrutinized. Despite the different lattice sites probed, $\delta\left(V_{\text {sample }}\right)$ is similar [Fig. 2(c)]. This observation indicates a preferred bond configuration that is achieved by relaxations of atom positions both at the tip apex and the graphene lattice and that is, therefore, a configuration adopted independent of the approach position. Selectively enhanced chemical reactivity of graphene $\mathrm{C}$ atoms on a metal surface were inferred previously from tunneling-to-contact transitions in STM junctions [43].

The density-functional and transport calculations (see Supplemental Material [11]) were carried out for a simplified quasi-one-dimensional Au tip on top of a free finite graphene sheet [Fig. 3(a)] [13,14]. They showed that the relaxation of the junction geometry prefers the top-C position to the hollow (by $\approx 0.2 \mathrm{eV}$ ) and bridge (by $\approx 0.04 \mathrm{eV}$ ) site of the graphene honeycomb cell. Consequently, independent of the tip position atop the graphene honeycomb cell, a bond configuration in which the tip-terminating $\mathrm{Au}$ atom is positioned atop a $\mathrm{C}$ atom is preferred. This result is consistent with the experimental finding of a site-independent variation of $\delta$ with $V_{\text {sample }}$ [Fig. 2(c)].

The atomic force induced by the applied field and the flowing current is calculated in the Born-Oppenheimer approximation and referred to as the bond force $F_{b}$ in the following. It is defined as the projection of $\mathbf{F}_{\mathrm{Au}}-\mathbf{F}_{\mathrm{C}}$ onto $\mathbf{r}_{\mathrm{Au}-\mathrm{C}}$, i.e., $F_{b}=\left(\mathbf{F}_{\mathrm{Au}}-\mathbf{F}_{\mathrm{C}}\right) \cdot \mathbf{r}_{\mathrm{Au}-\mathrm{C}} /\left|\mathbf{r}_{\mathrm{Au}-\mathrm{C}}\right|$, with $\mathbf{F}_{\mathrm{Au}}$ $\left(\mathbf{F}_{\mathrm{C}}\right)$ the total force acting on the $\mathrm{Au}(\mathrm{C})$ atom and $\mathbf{r}_{\mathrm{Au}-\mathrm{C}}=$ $\mathbf{r}_{\mathrm{C}}-\mathbf{r}_{\mathrm{Au}}$ the vector from $\mathrm{Au}$ to $\mathrm{C}$. The sign of $F_{b}$ is thus defined positive (negative) for attraction (repulsion).

In addition to the model setup [Fig. 3(a)] a $\mathrm{Au}-\mathrm{C}$ dimer was considered in the calculations. Both configurations reveal an unambiguous asymmetry of $F_{b}$ with $V_{\text {sample }}$ [Fig. 3(b)] -it is repulsive for $V_{\text {sample }}<0 \mathrm{~V}$ and attractive for $V_{\text {sample }}>0 \mathrm{~V}$. This behavior applies to different electron doping levels $n_{1}<n_{2}$, as well as to the presence or absence of a current $I$ across the junction. In particular, all models reveal similar $F_{b}$ magnitudes and a bond strengthening for positive $V_{\text {sample }}$, while deviations arise mainly due
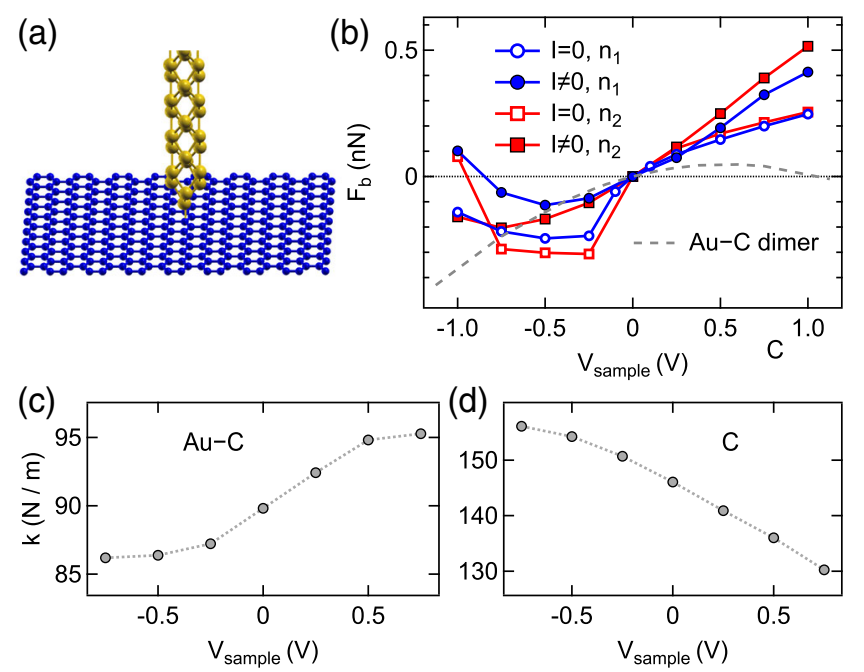

FIG. 3. (a) Model geometry comprising a quasi-one-dimensional $\mathrm{Au}$ tip and freestanding graphene. (b) Calculated bond force $F_{b}\left(V_{\text {sample }}\right)\left[F_{b}(0 \mathrm{~V})\right.$ set to $\left.0 \mathrm{nN}\right]$ for the model geometry in (a) (solid lines) and a $\mathrm{Au}-\mathrm{C}$ dimer (dashed line). The electric field is applied perpendicular to the graphene plane. Electron densities $n_{1}<n_{2}$ are adjusted by the Dirac point energy, $-400 \mathrm{meV}$ for $n_{1}$ and $-700 \mathrm{meV}$ for $n_{2}$. Solid (open) symbols reflect the presence (absence) of a current $I$. (c) Spring constant $k_{\mathrm{Au}-\mathrm{C}}$ as a function of $V_{\text {sample }}$ for doping $n_{1}$. (d) Spring constant $k_{\mathrm{C}}$ as a function of $V_{\text {sample }}$ calculated for displacing a $\mathrm{C}$ atom at doping $n_{1}$. 
to the different system sizes. For $V_{\text {sample }}>0 \mathrm{~V}$, the calculated data reveal a slightly larger $F_{b}$ for $n_{2}$ than for $n_{1}$. The higher $n$ doping yields a stronger screening in graphene, which tends to increase the voltage drop and local electric field at the $\mathrm{Au}-\mathrm{C}$ contact. This calculated result is compatible with the experimental observation of a more pronounced $\Delta f$ loop for $\mathrm{Li}$-intercalated graphene.

The sample voltage asymmetry of $F_{b}$ entails a corresponding asymmetry of the $\mathrm{Au}-\mathrm{C}$ bond strength, which is plotted as the $\mathrm{Au}-\mathrm{C}$ spring constant, $k_{\mathrm{Au}-\mathrm{C}}$, in Fig. 3(c). To obtain $k_{\mathrm{Au}-\mathrm{C}}$, the tip was displaced at constant $V_{\text {sample }}$ and the change in $F_{b}$ evaluated. The increased $k_{\mathrm{Au}-\mathrm{C}}$ at $V_{\text {sample }}>0 \mathrm{~V}$ supports the idea of graphene detachment upon tip retraction. The detachment scenario is further corroborated by the evolution of the spring constant of a graphene $\mathrm{C}$ atom, $k_{\mathrm{C}}$, with $V_{\text {sample }}$ [Fig. 3(d)] which was obtained by the finite displacement of the $\mathrm{C}$ atom along the surface normal. For increasing $V_{\text {sample }}, k_{\mathrm{C}}$ becomes significantly smaller. Therefore, the $\mathrm{C}$ atom is more easily moved due to the attraction to the $\mathrm{Au}$ atom for $V_{\text {sample }}>0 \mathrm{~V}$ than for $V_{\text {sample }}<0 \mathrm{~V}$.

An important question to be answered concerns the origin of the $V_{\text {sample }}$ asymmetry of $F_{b}$. The charge transfer in the $\mathrm{Au}-\mathrm{C}$ dimer [dots in Fig. 4(a)] as well as between the Au tip and graphene (squares) was calculated in a Hirshfeld charge analysis [44]. At $V_{\text {sample }}=0 \mathrm{~V}$, i.e., at zero electric field, electrons are transferred from (less electronegative) $\mathrm{Au}$ to (more electronegative) $\mathrm{C}$ of the dimer, leading to an electric dipole [45]. For $V_{\text {sample }}>0 \mathrm{~V}$ $\left(V_{\text {sample }}<0 \mathrm{~V}\right)$ electron transfer from Au to $\mathrm{C}$ is enhanced (reduced) compared to $0 \mathrm{~V}$. For the tip-graphene model, the electron transfer from the Au tip to graphene follows the same trend, with $V_{\text {sample }}>0 \mathrm{~V}$ supporting the electron transfer from the Au tip to graphene, which accumulates

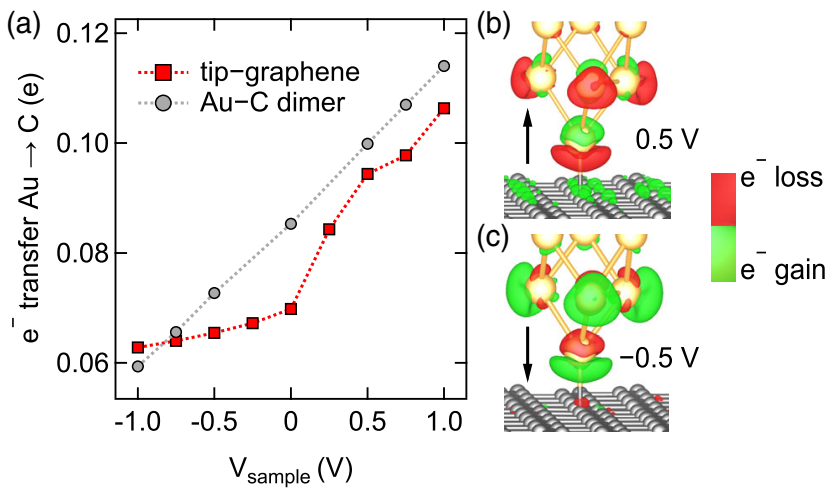

FIG. 4. (a) Hirshfeld charge analysis of electron transfer from $\mathrm{Au}$ to $\mathrm{C}$ depending on $V_{\text {sample }}$ calculated for the tip-graphene model (squares) and the $\mathrm{Au}-\mathrm{C}$ dimer (dots). (b) Induced electron density with respect to $0 \mathrm{~V}$ for the tip-graphene model at $V_{\text {sample }}=0.5 \mathrm{~V}$. Red (green) shaded areas depict electron loss (gain) with a density isosurface of $20 \mathrm{e} / \mathrm{nm}^{3}$ ( $e$, elementary charge). (c) Like (b), $V_{\text {sample }}=-0.5 \mathrm{~V}$. The arrow in (b) and (c) shows the electric-field direction. positive charge at the tip apex and negative charge in the atomic environment of the contacted graphene $\mathrm{C}$ atom [Fig. 4(b)]. The zero-bias dipole is enhanced and the polar bond is strengthened for $V_{\text {sample }}>0 \mathrm{~V}$. For $V_{\text {sample }}<0 \mathrm{~V}$, in contrast, electron transfer from $\mathrm{Au}$ to $\mathrm{C}$ is hindered and accumulates the opposite charge at the atoms [Fig. 4(c)]. The polar bond is thus weakened. This field-driven (rather than current-induced) effect is consistent with the experimentally observed nearly point-symmetric current with respect to $V_{\text {sample }}=0 \mathrm{~V}$ in a $V_{\text {sample }}$ interval where the asymmetry of $\delta$ is already clearly visible (see Supplemental Material [11]). Moreover, the calculated projected densities of states involving the relevant $p_{z}, d_{x z}, d_{y z}$ orbitals [11] clearly reveal the field-induced charge redistribution and show that positive (negative) $V_{\text {sample }}$ tends to decrease (increase) the $\mathrm{Au}-\mathrm{C}$ bond length [11]. This mechanism differs from the current-induced forces reported for homonuclear bonds [15,16,46,47], where changes in bond strength were attributed to modifications in the overlap population caused by the nonequilibrium filling of scattering states, rather than to charge transfer and dipole field interaction between the components.

In conclusion, the combination of atomic force microscopy and density-functional and nonequilibrium Green's function calculations unveils that the archetypical polar bond between two atoms can individually be influenced by the magnitude and orientation of an external electric field. The control of a single polar chemical two-atom bond proceeds via the field-induced charge transfer between the atoms with different electronegativity. Tailoring chemical-bond strengths at the single-atom level, together with the possibility of applying and releasing mechanical load, opens the path to locally distort matter and explore its response. From a chemical point of view, reactivity and catalytic activity may be accessed at the atomic scale with the presented methods.

Funding by the Deutsche Forschungsgemeinschaft (Grant No. KR 2912/10-3) and Villum Fonden (Grant No. 00013340) is acknowledged. The Center for Nanostructured Graphene $(\mathrm{CNG})$ is sponsored by the Danish Research Foundation (Grant No. DNRF103)

*slei@nanotech.dtu.dk joerg.kroeger@tu-ilmenau.de

[1] N. Xin, J. Guan, C. Zhou, X. Chen, C. Gu, Y. Li, M. A. Ratner, A. Nitzan, J. F. Stoddart, and X. Guo, Concepts in the design and engineering of single-molecule electronic devices, Nat. Rev. Phys. 1, 211 (2019).

[2] Y.F. Wang, J. Kröger, R. Berndt, H. Vázquez, M. Brandbyge, and M. Paulsson, Atomic-Scale Control of Electron Transport through Single Molecules, Phys. Rev. Lett. 104, 176802 (2010).

[3] G. Schull, T. Frederiksen, A. Arnau, D. Sanchez-Portal, and R. Berndt, Atomic-scale engineering of electrodes for single-molecule contacts, Nat. Nanotechnol. 6, 23 (2011). 
[4] L. Gross, F. Mohn, N. Moll, P. Liljeroth, and G. Meyer, The chemical structure of a molecule resolved by atomic force microscopy, Science 325, 1110 (2009).

[5] D. G. de Oteyza, P. Gorman, Y.-C. Chen, S. Wickenburg, A. Riss, D. J. Mowbray, G. Etkin, Z. Pedramrazi, H.-Z. Tsai, A. Rubio, M. F. Crommie, and F. R. Fischer, Direct imaging of covalent bond structure in single-molecule chemical reactions, Science 340, 1434 (2013).

[6] M. Emmrich, F. Huber, F. Pielmeier, J. Welker, T. Hofmann, M. Schneiderbauer, D. Meuer, S. Polesya, S. Mankovsky, D. Ködderitzsch, H. Ebert, and F. J. Giessibl, Subatomic resolution force microscopy reveals internal structure and adsorption sites of small iron clusters, Science 348, 308 (2015).

[7] F. Albrecht, N. Pavliček, C. Herranz-Lancho, M. Ruben, and J. Repp, Characterization of a surface reaction by means of atomic force microscopy, J. Am. Chem. Soc. 137, 7424 (2015).

[8] P. Hapala, R. Temirov, F. S. Tautz, and P. Jelínek, Origin of High-Resolution IETS-STM Images of Organic Molecules with Functionalized Tips, Phys. Rev. Lett. 113, 226101 (2014).

[9] F. Albrecht, J. Repp, M. Fleischmann, M. Scheer, M. Ondráček, and P. Jelínek, Probing Charges on the Atomic Scale by Means of Atomic Force Microscopy, Phys. Rev. Lett. 115, 076101 (2015).

[10] M. Ellner, N. Pavliček, P. Pou, B. Schuler, N. Moll, G. Meyer, L. Gross, and R. Peréz, The electric field of CO tips and its relevance for atomic force microscopy, Nano Lett. 16, 1974 (2016).

[11] See Supplemental Material at http://link.aps.org/supplemental/ 10.1103/PhysRevLett.126.216801 for experimental details on sample and tip preparation, as well as data analysis tools, vertical-distance evolution of the current (Figs. S1 and S2), reversibility of chemical-bond formation (Fig. S3), currentvoltage characteristics (Fig. S4), theoretical details, calculated projected densities of states (Fig. S5) and calculated total energies (Fig. S6), which includes Refs. [12-31].

[12] M. Omidian, N. Néel, E. Manske, J. Pezoldt, Y. Lei, and J. Kröger, Structural and local electronic properties of clean and Li-intercalated graphene on SiC(0001), Surf. Sci. 699, 121638 (2020).

[13] G. Calogero, N. Papior, M. Koleini, M. H. L. Larsen, and M. Brandbyge, Multi-scale approach to first-principles electron transport beyond $100 \mathrm{~nm}$, Nanoscale 11, 6153 (2019).

[14] N. Papior, G. Calogero, S. Leitherer, and M. Brandbyge, Removing all periodic boundary conditions: Efficient nonequilibrium Green's function calculations, Phys. Rev. B 100, 195417 (2019).

[15] S. Leitherer, N. Papior, and M. Brandbyge, Current-induced atomic forces in gated graphene nanoconstrictions, Phys. Rev. B 100, 035415 (2019).

[16] J. Kröger, N. Néel, T. O. Wehling, and M. Brandbyge, Local probes of graphene lattice dynamics, Small Methods 4, 1900817 (2020).

[17] F. J. Giessibl, High-speed force sensor for force microscopy and profilometry utilizing a quartz tuning fork, Appl. Phys. Lett. 73, 3956 (1998).

[18] L. Limot, J. Kröger, R. Berndt, A. Garcia-Lekue, and W. A. Hofer, Atom Transfer and Single-Adatom Contacts, Phys. Rev. Lett. 94, 126102 (2005).
[19] J. Kröger, N. Néel, and L. Limot, Contact to single atoms and molecules with the tip of a scanning tunnelling microscope, J. Phys. Condens. Matter 20, 223001 (2008).

[20] R. Berndt, J. Kröger, N. Néel, and G. Schull, Controlled single atom and single molecule contacts, Phys. Chem. Chem. Phys. 12, 1022 (2010).

[21] I. Horcas, R. Fernández, J. M. Gómez-Rodríguez, J. Colchero, J. Gómez-Herrero, and A. M. Baro, WSXM: A software for scanning probe microscopy and a tool for nanotechnology, Rev. Sci. Instrum. 78, 013705 (2007).

[22] N. Néel, J. Kröger, and R. Berndt, Quantized Conductance of a Single Magnetic Atom, Phys. Rev. Lett. 102, 086805 (2009).

[23] N. Néel, J. Kröger, and R. Berndt, Two-level conductance fluctuations of a single-molecule junction, Nano Lett. 11, 3593 (2011).

[24] J. M. Soler, E. Artacho, J. D. Gale, A. García, J. Junquera, P. Ordejón, and D. Sánchez-Portal, The SIESTA method for ab initio order-N materials simulation, J. Phys. Condens. Matter 14, 2745 (2002).

[25] M. Brandbyge, J.-L. Mozos, P. Ordejón, J. Taylor, and K. Stokbro, Density-functional method for nonequilibrium electron transport, Phys. Rev. B 65, 165401 (2002).

[26] N. Papior, N. Lorente, T. Frederiksen, A. García, and M. Brandbyge, Improvements on non-equilibrium and transport Green function techniques: The next-generation transiesta, Comput. Phys. Commun. 212, 8 (2017).

[27] N. Papior, SISL, v0.9.4: Toolbox for electronic structure calculations, http://zerothi.github.io/sisl/docs/latest/index.html (2018).

[28] J. P. Perdew, K. Burke, and M. Ernzerhof, Generalized Gradient Approximation Made Simple, Phys. Rev. Lett. 77, 3865 (1996).

[29] J. Halle, N. Néel, M. Fonin, M. Brandbyge, and J. Kröger, Understanding and engineering phonon-mediated tunneling into graphene on metal surfaces, Nano Lett. 18, 5697 (2018).

[30] N. Papior, T. Gunst, D. Stradi, and M. Brandbyge, Manipulating the voltage drop in graphene nanojunctions using a gate potential, Phys. Chem. Chem. Phys. 18, 1025 (2016).

[31] N. M. Caffrey, L. I. Johansson, C. Xia, R. Armiento, I. A. Abrikosov, and C. Jacobi, Structural and electronic properties of Li-intercalated graphene on $\mathrm{SiC}(0001)$, Phys. Rev. B 93, 195421 (2016).

[32] C. Chang, I. Tsong, Y. Wang, and R. Davis, Scanning tunneling microscopy and spectroscopy of cubic $\beta$-SiC(111) surfaces, Surf. Sci. 256, 354 (1991).

[33] S. Kim, J. Ihm, H. J. Choi, and Y.-W. Son, Origin of Anomalous Electronic Structures of Epitaxial Graphene on Silicon Carbide, Phys. Rev. Lett. 100, 176802 (2008).

[34] A. T. N'Diaye, J. Coraux, T. N. Plasa, C. Busse, and T. Michely, Structure of epitaxial graphene on $\operatorname{Ir}(111)$, New J. Phys. 10, 043033 (2008).

[35] C. Virojanadara, S. Watcharinyanon, A. A. Zakharov, and L. I. Johansson, Epitaxial graphene on $6 \mathrm{H}-\mathrm{SiC}$ and $\mathrm{Li}$ intercalation, Phys. Rev. B 82, 205402 (2010).

[36] S. Fiori, Y. Murata, S. Veronesi, A. Rossi, C. Coletti, and S. Heun, Li-intercalated graphene on SiC(0001): An STM study, Phys. Rev. B 96, 125429 (2017). 
[37] F. J. Giessibl, A direct method to calculate tip-sample forces from frequency shifts in frequency-modulation atomic force microscopy, Appl. Phys. Lett. 78, 123 (2001).

[38] J. E. Sader and S. P. Jarvis, Accurate formulas for interaction force and energy in frequency modulation force spectroscopy, Appl. Phys. Lett. 84, 1801 (2004).

[39] J. E. Sader, B. D. Hughes, F. Huber, and F. J. Giessibl, Interatomic force laws that evade dynamic measurement, Nat. Nanotechnol. 13, 1088 (2018).

[40] T. Mashoff, M. Pratzer, V. Geringer, T. J. Echtermeyer, M. C. Lemme, M. Liebmann, and M. Morgenstern, Bistability and oscillatory motion of natural nanomembranes appearing within monolayer graphene on silicon dioxide, Nano Lett. 10, 461 (2010).

[41] N. N. Klimov, S. Jung, S. Zhu, T. Li, C. A. Wright, S. D. Solares, D. B. Newell, N. B. Zhitenev, and J. A. Stroscio, Electromechanical properties of graphene drumheads, Science 336, 1557 (2012).
[42] M. Wolloch, G. Feldbauer, P. Mohn, J. Redinger, and A. Vernes, $\mathrm{Ab}$ initio calculation of the real contact area on the atomic scale, Phys. Rev. B 91, 195436 (2015).

[43] S. J. Altenburg, J. Kröger, B. Wang, M.-L. Bocquet, N. Lorente, and R. Berndt, Graphene on $\mathrm{Ru}(0001)$ : Contact Formation and Chemical Reactivity on the Atomic Scale, Phys. Rev. Lett. 105, 236101 (2010).

[44] F. L. Hirshfeld, Bonded-atom fragments for describing molecular charge densities, Theor. Chim. Acta 44, 129 (1977).

[45] M. Rahm, T. Zeng, and R. Hoffmann, Electronegativity seen as the ground-state average valence electron binding energy, J. Am. Chem. Soc. 141, 342 (2019).

[46] M. Brandbyge, K. Stokbro, J. Taylor, J.-L. Mozos, and P. Ordejón, Origin of current-induced forces in an atomic gold wire: A first-principles study, Phys. Rev. B 67, 193104 (2003).

[47] J. Brand, S. Leitherer, N. R. Papior, N. Néel, Y. Lei, M. Brandbyge, and J. Kröger, Nonequilibrium bond forces in single-molecule junctions, Nano Lett. 19, 7845 (2019). 